\title{
Running Characteristics of Aerodynamic Bearing with Self-Lifting Capability at Low Rotational Speed
}

\author{
Tadeusz Adam Stolarski \\ Mechanical Engineering, School of Engineering and Design, Brunel University, Uxbridge, Middlesex UB8 3PH, UK \\ Correspondence should be addressed to Tadeusz Adam Stolarski, mesttas@brunel.ac.uk
}

Received 25 June 2010; Revised 11 January 2011; Accepted 9 February 2011

Academic Editor: M. M. Khonsari

Copyright (๑) 2011 Tadeusz Adam Stolarski. This is an open access article distributed under the Creative Commons Attribution License, which permits unrestricted use, distribution, and reproduction in any medium, provided the original work is properly cited.

\begin{abstract}
An aerodynamic journal bearing that is capable of self-generating squeeze-film pressure is presented and its dynamic characteristics investigated numerically and experimentally. A numerical method based on a time-marching static model was applied to assess the orbit trajectory path of the rotor upon a perturbation. Experimental results were obtained to validate the effect of the selfgenerated squeeze-film pressure on the stability of the rotor. Analyzing the Fast Fourier Transform (FFT) responses of the rotor orbits enabled identification of self-excited whirling instabilities. Both numerical and experimental results showed that increasing the squeeze-film effect of the bearing could raise the threshold speed of instability.
\end{abstract}

\section{Introduction}

Operational speed of gas bearings is limited by instabilities associated with modal vibration and self-excited whirl of the rotor. The latter is often considered to be the more destructive of the two. Reynolds and Gross [1] experimented on self-excited whirl of an aerodynamic journal bearing by increasing the speed until whirling instability was observed from the orbital path. It was found that an indication of a developing self-excited whirl is a tendency of the whirl circle to break into two connecting circles. It was also concluded that running at high eccentricities either with sufficient loading or imbalance of rotor can raise the instability threshold. More recent techniques like Fast Fourier Transform (FFT) [2] and bifurcation analysis have been applied to rotor orbits in determining the dynamic characteristics of the bearing.

Numerical techniques have been applied for the assessment of the dynamic performance and stability of fluidfilm bearings. The linear perturbation method calculates the linear stiffness and damping coefficients from a small distance away from the rotor's equilibrium position [3]. Using those coefficients, Lund [4] introduced the critical mass parameter. The critical mass is the threshold mass to which the rotor can operate stably, and consequently, negative critical mass suggests the inevitability of selfexcited whirl instability. The flexibility of the nonlinear orbit method, introduced by Castelli and Elrod [5], has been recognized owing to the growth of computational power. The orbit method consists of coupling the motion and fluid equations and marching them together in time. If the journal is displaced from its equilibrium position, the orbital path of the rotor is used to assess the stability of the bearing. If the rotor returns to the equilibrium position, the condition is considered stable, and unstable if the journal spirals out with increasing radius. Although the nonlinear orbit method has no limitations or assumptions, the cost of the process is still considered to be high as compared to other analytical methods, particularly for parametric studies. Dynamics of hydrostatic micro-gas journal bearing was studied by Liu and Spakovszky [6]. In particular, they considered the effect of bearing stiffness anisotropy on bearing's dynamic behaviour. In another paper by Teo et al. [7], dynamics of ultra-short gas bearing is presented and discussed.

In the previous paper, the authors proposed an aerodynamic journal bearing with elastic hinges that utilizes piezoelectric elements to actively deform the plain circular bearing clearance to a three-lobe bearing clearance [8]. Experimental and numerical results show that the bearing was capable of generating the squeeze-film pressure effect to 
TABle 1: Dimensions of test bearing.

\begin{tabular}{lc}
\hline Variable & Values \\
\hline$m_{0}$ & $650 \mathrm{~g}$ \\
$l_{0}$ & $25 \mathrm{~mm}$ \\
$r_{0}$ & $15 \mathrm{~mm}$ \\
$c$ & $40 \mu \mathrm{m}$ \\
$e_{w 0}$ & $1.77 \mu \mathrm{m}(60 \mathrm{~V}$ offset $)$ \\
\hline
\end{tabular}

levitate the rotor. This self-generating squeeze-film pressure was proposed as a substitute to aerostatic pressure. The dynamic response of a rotor supported by squeeze-film pressure at low rotational speeds is the main theme of this paper. Experimental and numerical results are presented to demonstrate the role of the self-generated squeeze-film pressure in improving the dynamic characteristics of the gas bearing. It is believed that for the first time this paper demonstrates dynamic behaviour of the rotor supported purely by the pressure generated due to squeeze-film mechanism at rotational speeds of up to the initiation of aerodynamic pressure generation.

\section{Theoretical Analyses}

The working principle of the bearing has been described elsewhere [6] and shall not be repeated here. Figure 1(a) shows the three-lobe test bearing and the dimensions are summarized in Table 1. Figure 1(b) shows schematic diagram of the shaft and the bearing; the bearing is preloaded by the piezoelectric actuators to create the static three-lobe bearing clearance. The active three-lobe film thickness [6] is described as follows:

$$
\begin{aligned}
H_{W}= & \frac{h_{W O}}{c}+\frac{h_{W A}}{c} \\
= & 1+\varepsilon_{Y} \cos (\theta)+\varepsilon_{X} \sin (\theta)-\varepsilon_{W O} \cos (3 \theta) \\
& +\left|0.07986 \varepsilon_{W O} \cos (3 \theta)\right|-\varepsilon_{W A} \sin (\omega t) \cos (3 \theta) \\
& +\left|0.07986 \varepsilon_{W A} \sin (\omega t) \cos (3 \theta)\right| .
\end{aligned}
$$

A full transient analysis of the rotor and the journal bearing involves two squeeze-film effects, one due to the constant cyclic squeezing action of the bearing generated by the piezoelectric actuators and the other due to the natural translation of the rotor.

Consideration of both squeeze terms yields to a highly nonlinear transient problem, with an increase of computational time and instabilities. For this reason, the full transient method proposed by Castelli and Elrod [5] had never been applied to squeeze-film air bearings. However, by assuming the rotor translation speed to be negligible compared to the bearing's active squeeze-film frequency the latter squeeze term may be neglected and a quasistatic method developed.

2.1. Comparison between the Static and Transient Orbit Method. The comparison between the static and transient orbit method was investigated on the plain, three-wave aerodynamic bearing. Here, the self-generated squeeze-film frequency is zero, $\varepsilon_{W A}=0, \omega=0$. The governing equations for the transient and static orbit method are shown in (2a) and (2b), respectively; details of solution procedures are shown in the next section. The static method is different to the transient method by neglecting the natural squeeze term in the Reynolds equation, and consequently the rotor's translational inertia was also neglected. However, the solution procedure is the same for both methods when an attempt is made to search for the equilibrium position by a time-marching technique. Although the true rotor trajectory path may only be obtained from the full transient solutions, if stable, it will yield to the same steady-state solution as the static analysis.

$$
\begin{gathered}
\frac{\partial}{\partial \theta}\left(P H^{3} \frac{\partial P}{\partial \theta}\right)+\frac{\partial}{\partial Z}\left(P H^{3} \frac{\partial P}{\partial Z}\right)=\Lambda \frac{\partial(P H)}{\partial \theta}+2 \Lambda \frac{\partial(P H)}{\partial T} \\
\dot{\varepsilon}_{Y}=\ddot{\varepsilon}_{Y 0} \Delta T+\dot{\varepsilon}_{Y 0}, \quad \dot{\varepsilon}_{X}=\ddot{\varepsilon}_{X 0} \Delta T+\dot{\varepsilon}_{X 0} \\
\varepsilon_{Y}=\dot{\varepsilon}_{Y} \Delta T+\varepsilon_{Y 0}, \quad \varepsilon_{X}=\dot{\varepsilon}_{X} \Delta T+\varepsilon_{X 0} \\
\frac{\partial}{\partial \theta}\left(P H^{3} \frac{\partial P}{\partial \theta}\right)+\frac{\partial}{\partial Z}\left(P H^{3} \frac{\partial P}{\partial Z}\right)=\Lambda \frac{\partial(P H)}{\partial \theta} \\
\dot{\varepsilon}_{Y}=\ddot{\varepsilon}_{Y 0} \Delta T, \quad \dot{\varepsilon}_{X}=\ddot{\varepsilon}_{X 0} \Delta T \\
\varepsilon_{Y}=\dot{\varepsilon}_{Y} \Delta T+\varepsilon_{Y 0}, \quad \varepsilon_{X}=\dot{\varepsilon}_{X} \Delta T+\varepsilon_{X 0}
\end{gathered}
$$

Figures 2(a)-2(c) show the orbital paths of the rotor obtained from the static and transient method for a plain circular journal bearing with increasing external load. Remarkable differences in the two trajectories can be seen, in particular at the presence of self-excited whirl. The transient solution of Figure 2(a) showed an increasing whirling orbital path, whereas the static solution showed a converging solution. Figures $2(a)-2(c)$ showed that the stability of the rotor could be improved by increasing the external load; here the static and transient analysis showed fast convergence of the same solution.

Figures 3(a)-3(d) show the effect of the rotor's trajectory paths with the size of the static three-lobe clearance. With a light load of $56.65 \mathrm{~g}$ and small static amplitude $\left(\varepsilon_{w o}\right)$, the results showed that the rotor's equilibrium position was never reached, and instead, the rotor undergoes a periodic orbit as shown in Figures 3(a) and 3(b). However, as a result of neglecting the rotor's inertia, the sizes of the orbits obtained from the static solution are much smaller than those from the transient solution. Figures 3(c) and 3(d) showed that the stability of the plain three-lobe bearing could be improved by increasing the lobe eccentricity. Here the static and transient solutions showed good agreements with the absence of whirling.

Although the trajectory paths obtained from the static analysis differ from those produced by the true transient analysis, they might still be applied to assess the dynamic characteristic of the bearing. If the bearing is operating stable, the static solutions should yield to a converged value; however, if excess oscillations are found, self-excited 


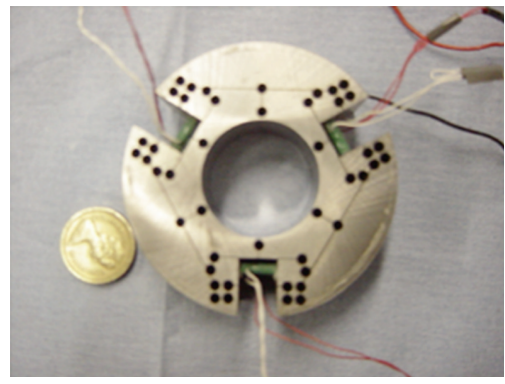

(a)

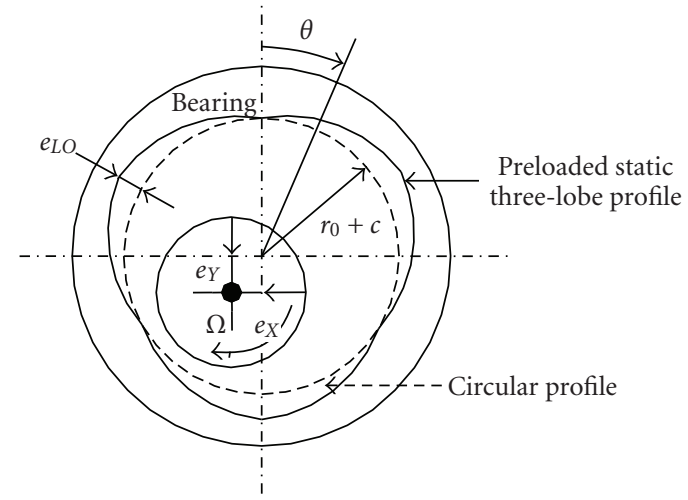

(b)

Figure 1: (a) Photograph of the test bearing shell with arrangements of piezoelectric actuators. (b) The static three-lobe geometry of the bearing (not to scale).

whirl instability is most likely to occur. Self-excited whirling is commonly found in lightly loaded bearings with small eccentricities $\varepsilon_{Y}$. However, it has to be emphasized that the correct method is the true transient orbit method even though it is time consuming.

2.2. Application of the Static Orbit Method. With the assumptions generally used in gas film bearing analysis [6], the pressure of the film can be obtained by solving the quasisteady-state Reynolds equation, as shown in (3) in its nondimensional form.

$$
\frac{\partial}{\partial X}\left(P H^{3} \frac{\partial P}{\partial X}\right)+\frac{\partial}{\partial Z}\left(P H^{3} \frac{\partial P}{\partial Z}\right)=\Lambda \frac{\partial(P H)}{\partial X}+\sigma \frac{\partial(P H)}{\partial \tau} .
$$

The squeeze number $\sigma$ is given by

$$
\sigma=\frac{12 \mu \omega r_{0}^{2}}{p_{a} c^{2}}
$$

and the bearing number $\Lambda$ is represented by

$$
\Lambda=\frac{12 \mu \Omega \tau_{0}}{p_{0} c^{2}} .
$$

Here $\Lambda$ characterizes the self-acting aerodynamic pressure generated by the rotational speed $\Omega$ of the rotor at an eccentric position and $\sigma$ characterizes the self-acting squeeze-film pressure generated by cycling three-lobe bearing clearance at a frequency of $\omega$.

The nonlinear Reynolds equation was discretised using the Finite Difference Method (FDM) and solved iteratively using an under-relaxation technique with the following boundary conditions.

$$
\left.P\right|_{z=0}=\left.P\right|_{z=1}=1 .
$$

The initial conditions are

$$
\left.P\right|_{\tau=0}=1,\left.\quad \varepsilon_{y}\right|_{\tau=0}=\left.\varepsilon_{Y 0}\right|_{\tau=0}=\left.\varepsilon_{X=0}\right|_{\tau=0}=0 .
$$

For a continuous film,

$$
\left.\left.P\right|_{\theta=0} P\right|_{\theta=2 \pi} .
$$

For constant periodicity at quasisteady-state,

$$
\left.P\right|_{\tau}=\left.P\right|_{\tau+2 \pi},\left.\quad H\right|_{\tau}=\left.H\right|_{\tau+2 \pi} .
$$

The forces arising from the pressure in the air film were estimated as the integrals of the pressure over the spatial domains for a complete cycle (see (10a) and (10b)). Then the timed averaged of the pressure forces was estimated for one cycle as shown in (11a) and (11b). This time averaged force is the load-carrying capacity of squeeze-film air bearings.

$$
\begin{gathered}
F_{P Y}=-\int_{0}^{1} \int_{0}^{2 \pi} P \cos (\theta) d \theta d Z, \\
F_{P X}=-\int_{0}^{1} \int_{0}^{2 \pi} P \sin (\theta) d \theta d Z, \\
\bar{F}_{P Y}=\frac{1}{2 \pi} \int_{0}^{2 \pi} F_{P Y} d \tau, \\
\bar{F}_{P X}=\frac{1}{2 \pi} \int_{0}^{2 \pi} F_{P X} d \tau .
\end{gathered}
$$

The equations of motion for a perfectly balanced, rigid rotor in the $y$ and $x$ direction are shown in (12a) and (12b), respectively,

$$
\begin{gathered}
M_{0} \ddot{\varepsilon}_{Y}=\bar{F}_{P Y}+W_{Y}, \\
M_{0} \ddot{\varepsilon}_{X}=\bar{F}_{P X},
\end{gathered}
$$




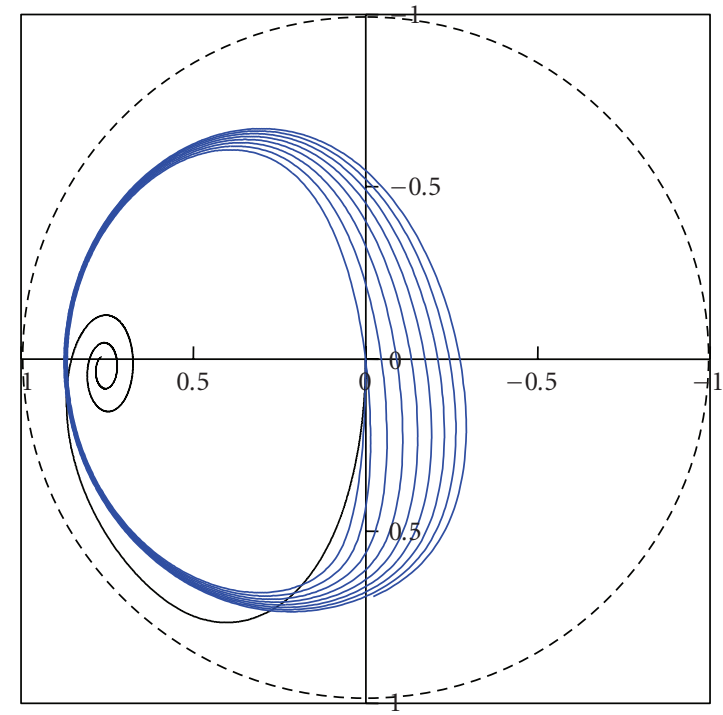

(a)

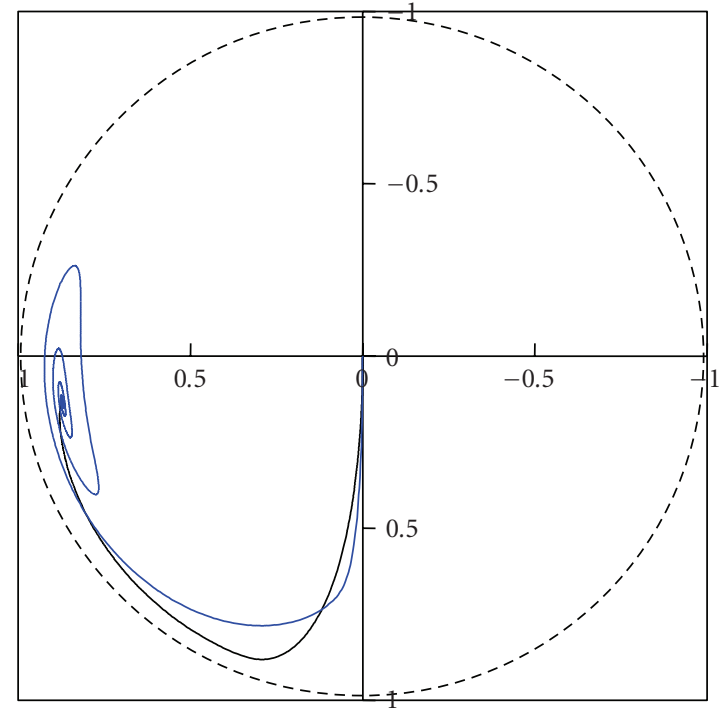

(b)

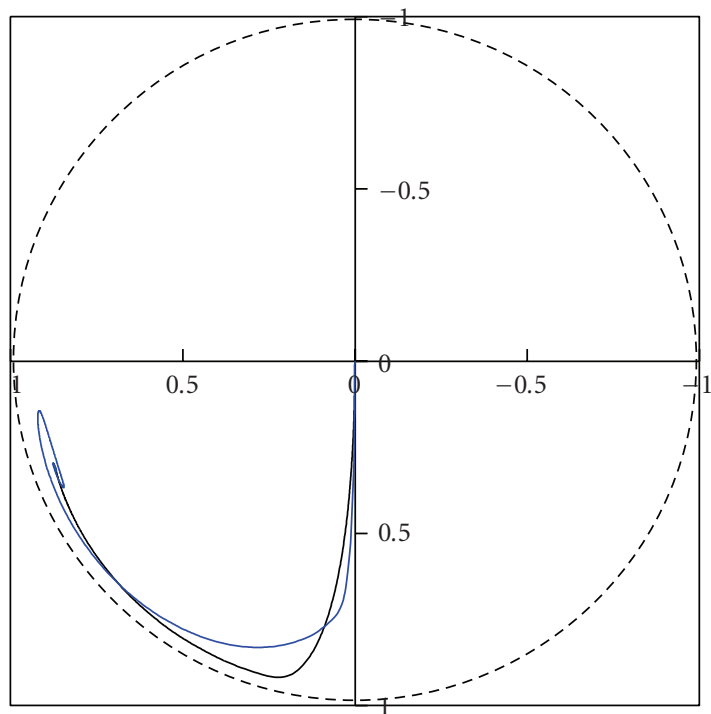

Static
Transient

(c)

Figure 2: Trajectories path of rotor's center (expressed as a ration of rotor centre position over bearing clearance), comparison between transient and static results for plain circular journal bearing, $\Omega=1000 \mathrm{rpm}, c=40 \mu \mathrm{m}, l_{0}=25 \mathrm{~mm}$, and $r_{0}=15 \mathrm{~mm}$, with loads of (a) $56.65 \mathrm{~g}$, (b) $113.4 \mathrm{~g}$, and (c) $170.2 \mathrm{~g}$.

where the dimensionless quantities of mass and external gravitational load are shown in the following:

$$
\begin{gathered}
M_{0}=\frac{m_{0} c \Omega^{2}}{p_{0} r_{0}^{2}}, \\
W_{Y}=\frac{m_{0} g}{p_{0} r_{0}^{2}} .
\end{gathered}
$$

By coupling the Reynolds equation and the equations of motion, the quasisteady-state position was solved iteratively using the Euler method. Firstly, the acceleration due to unbalanced forces was obtained by solving the equations of motion. Then the velocities and displacements were estimated from (14a) and (14b), respectively, in which the subscripts $Y 0$ and $X 0$ denote values from the previous time step. The Eulerian time step $\Delta T$ does not need to be as small as $\Delta \tau$ but, more conveniently, it is often related to rotor rotational speed rather than squeeze-film frequency, $\Delta T(\Omega)>\Delta \tau(\omega)$.

$$
\begin{gathered}
\dot{\varepsilon}_{Y}=\ddot{\varepsilon}_{Y} \Delta T, \quad \dot{\varepsilon}_{X}=\ddot{\varepsilon}_{X} \Delta T, \\
\varepsilon_{Y}=\dot{\varepsilon}_{Y} \Delta T+\varepsilon_{Y 0}, \quad \varepsilon_{X}=\dot{\varepsilon}_{X} \Delta T+\varepsilon_{X 0} .
\end{gathered}
$$




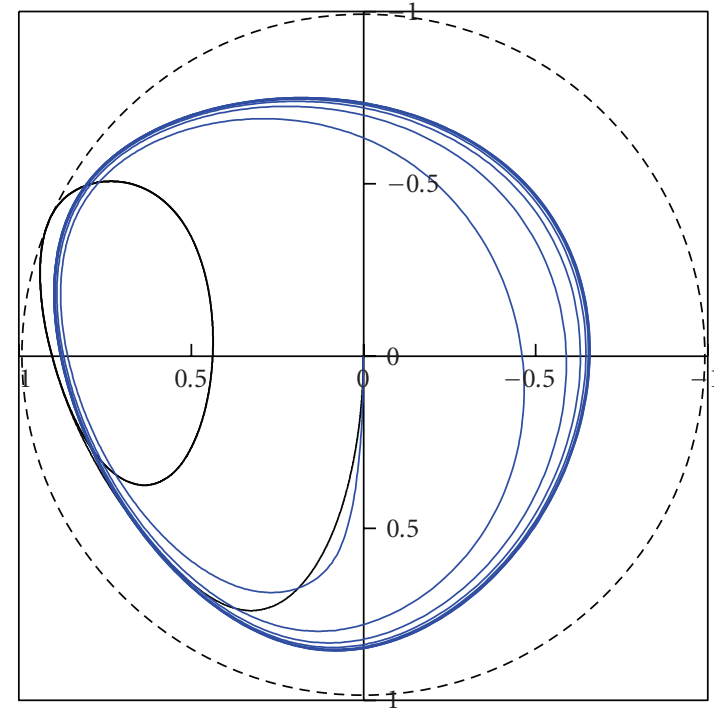

(a)

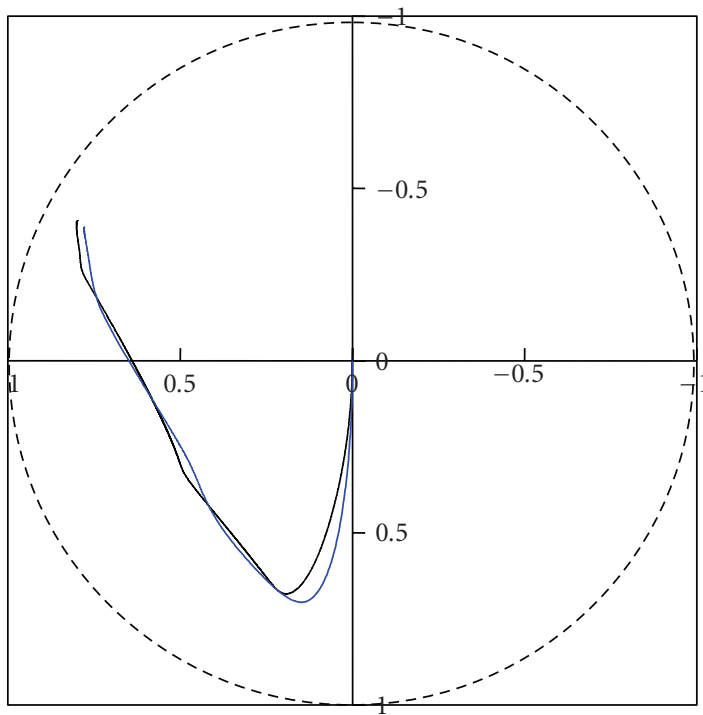

- Static
Transient

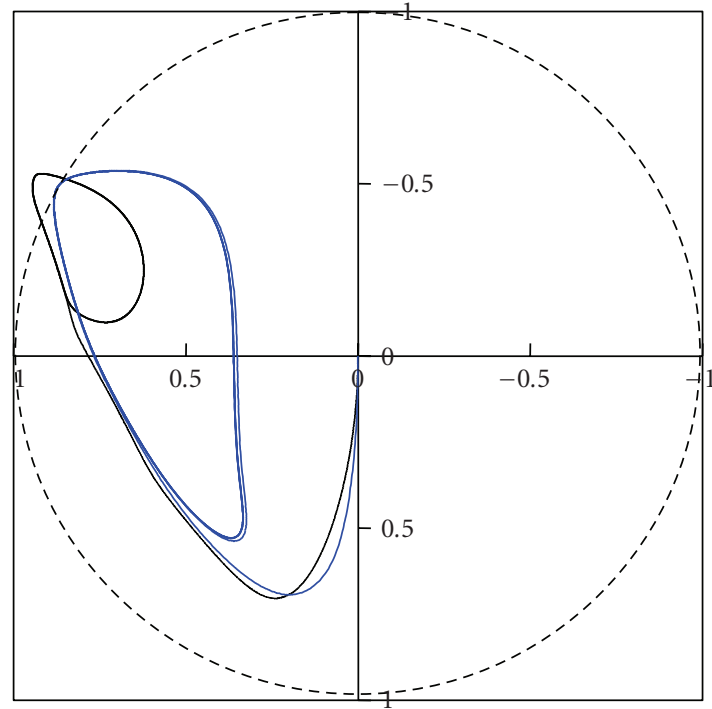

(b)

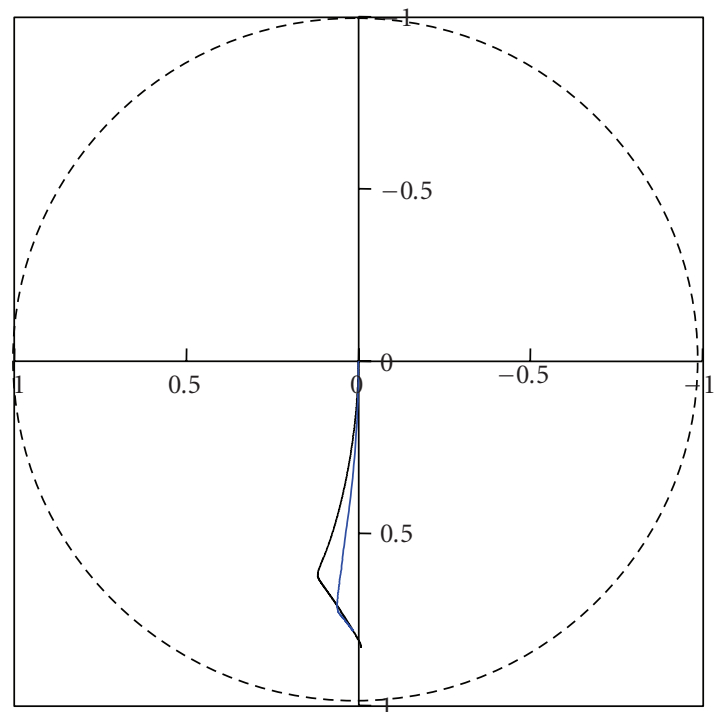

Static
Transien

(c)

(d)

FIGURE 3: Trajectories path of rotor center (expressed as a ration of rotor centre position over bearing clearance), comparison between transient and static results for plain three-lobe bearings with load of $56.65 \mathrm{~g}, \Omega=1000 \mathrm{rpm}, c=40 \mu \mathrm{m}, l_{0}=25 \mathrm{~mm}$, and $r_{0}=15 \mathrm{~mm}$ and lobe eccentricities $\left(\varepsilon_{W 0}\right)$ of (a) 0.0875 , (b) 0.25 , and (c) 0.375 , (d) 0.5 .

With the new rotor position, the next film pressure cycle was solved again using the Reynolds' equation. This procedure is repeated until convergence is achieved, where the rotor eccentricities $\varepsilon_{Y}$ and $\varepsilon_{Y}$ converge to a steady-state solution for one cycle.

\section{Experimental Apparatus and Procedure}

The test rig, consisting of the housing for the bearing and the rotor, is shown in Figure 4.
The rotor is supported axially by an aerostatic thrust bearing and in the radial direction by the test bearing. Prior to testing, the compressible squeeze-film effect was generated by piezoelectric actuators to levitate the rotor from the test bearing. The apparatus used to drive the piezoelectric actuators consists of a function generator and an amplifier [6]. The photograph of the test rig and the apparatus is shown in Figure 5. The voltage waveform to the actuators controlled the compressible squeeze-film effect. The rotor was driven by an air turbine system, and an optical sensor monitored the rotational speed of the rotor. 


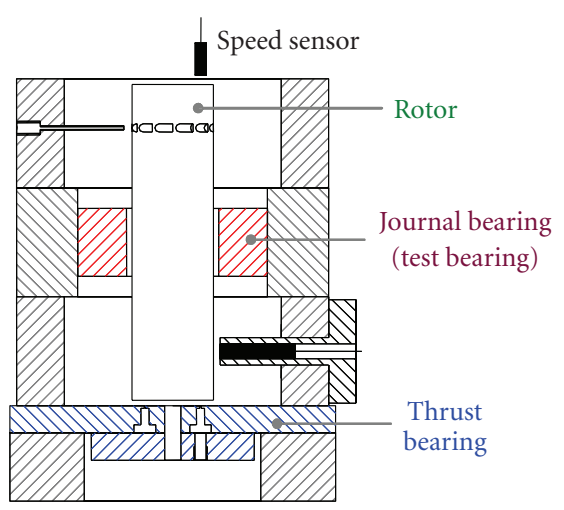

(a)

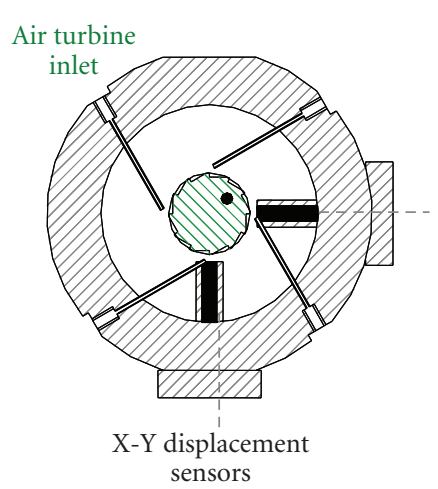

(b)

FIgURE 4: Schematic of the experimental test rig.

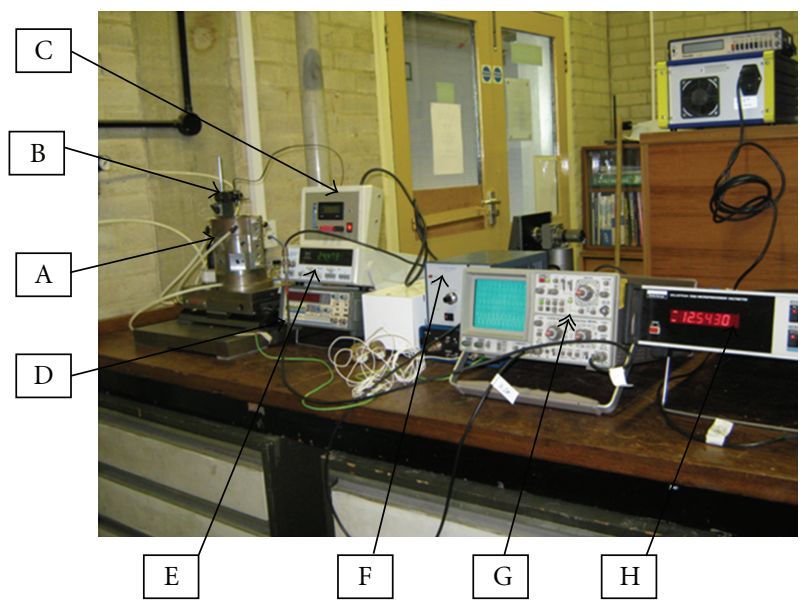

Figure 5: Overview of experimental test rig and apparatus: (A) Test Rig, (B) Speed Optical Sensor, (C) Speed Meter, (D) Displacement Probe Actuator, (E) Sine Wave Generator, (F) Piezoelectric Amplifier, (G) Oscilloscope, (H) Voltmeter.

It was found that when the squeeze-film action was activated, little or no frictional contact existed between the rotor and the test bearing, and the rotor was then driven with very little effort by the pressurized air jet (air turbine). Two capacitance displacement sensors, mounted in an X-Y configuration, monitor the rotor translation motion. All test data are monitored and saved using a computer-based data acquisition system.

Fast Fourier Transform (FFT) of the rotor's X and Y amplitudes shows the behavior of the rotor vibration. The magnitude and frequency of the vibration was summarized by the magnitude of the peaks on the frequency spectrum. Peaks below the rotational frequency showed subsynchronous whirling and the peak at the rotational frequency shows the unbalance response of the rotor. Subsynchronous whirling of the rotor is often shown as evident of self-excited instability [1], the mode of whirl may be translational, conical, or a combination of both [1].
TABLE 2: Test parameters for experimental and numerical studies.

\begin{tabular}{lcc}
\hline Variable & Settings & Values \\
\hline$m_{\alpha}$ & $0^{\circ}, 5^{\circ}, 10^{\circ}, 20^{\circ}$ tilt & $0 \mathrm{~g}, 56.65 \mathrm{~g}, 113.4 \mathrm{~g}, 170.2 \mathrm{~g}, 222.3 \mathrm{~g}$ \\
$e_{w}$ & $30 \mathrm{~V}, 40 \mathrm{~V}, 50 \mathrm{~V}$ & $0.45 \mu \mathrm{m}, 0.65 \mu \mathrm{m}, 0.85 \mu \mathrm{m}$ \\
$\omega / 2 \pi$ & $1500 \mathrm{~Hz}$ & $1500 \mathrm{~Hz}$ \\
\hline
\end{tabular}

The whole test rig was clamped to a tilting base. By tilting the test rig to an inclined angle, loading to the test bearing was provided by the gravitational force of the rotor. The load on the bearing was calculated from the expression: $W=m_{0} g \sin \alpha$.

It was found that although the test bearing was capable of supporting a load of $222.3 \mathrm{~g}$ corresponding to $20^{\circ}$ tilt [6], the large steady state eccentricities $\left(\varepsilon_{Y}>0.9\right)$ indicated that the practical maximum static load of the bearing is $56.65 \mathrm{~g}$ $\left(5^{\circ}\right.$ tilt). It was also found that the effect was governed by $e_{W}$ and $\omega$. For the range tested, results showed that $e_{W}$ has more pronounce effect at nonresonance frequencies. For this study, the squeeze-film effect was investigated by varying $e_{W}$ as summarized in Table 2.

\section{Results and Discussions}

Figures $6(a)-6(c)$ show the numerical results of the rotor trajectory paths using the static analysis for a load of $56.65 \mathrm{~g}$. It can be seen that with the absence of the self-generated squeeze-film effect, the rotor will undergo periodic orbits and possibly whirling which points to its instability. For this reason, the self-generating squeeze-film effect is necessary not only to levitate the rotor at start up and coast down stages, but also in operation, where the rotor is driven to speed.

Figure 6(c) shows that for a load of $56.65 \mathrm{~g}$ (rather light load) the rotor is limited to speed of $1020 \mathrm{rpm}$ where selfexcited whirling is inevitable. Stability of the rotor can be improved by increasing the load on the bearing or by increasing the magnitude of pressure generated by the squeezefilm mechanism. The results show that the squeeze-film 


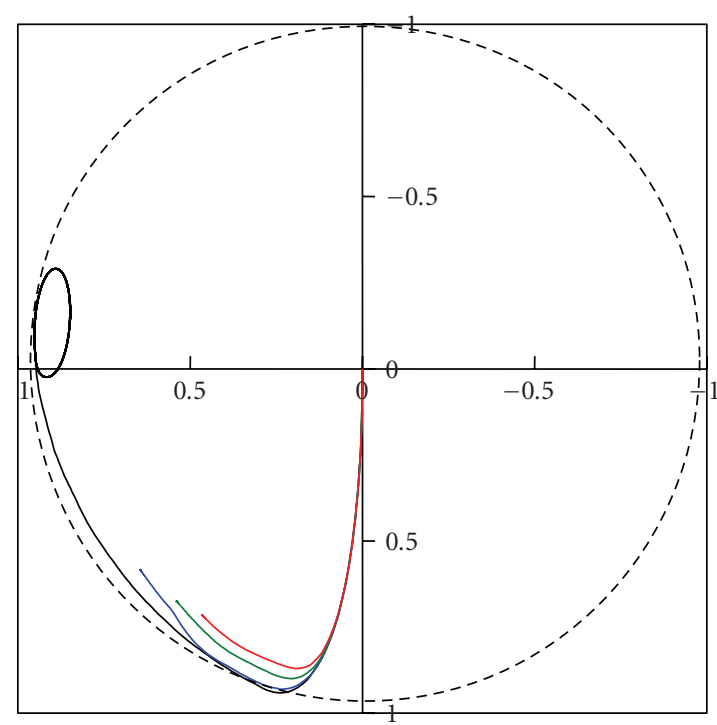

(a)

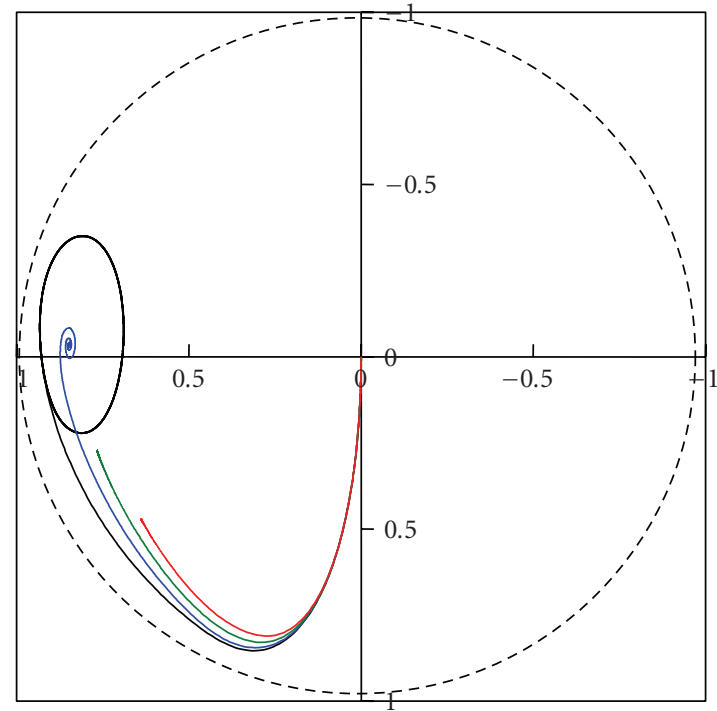

(b)

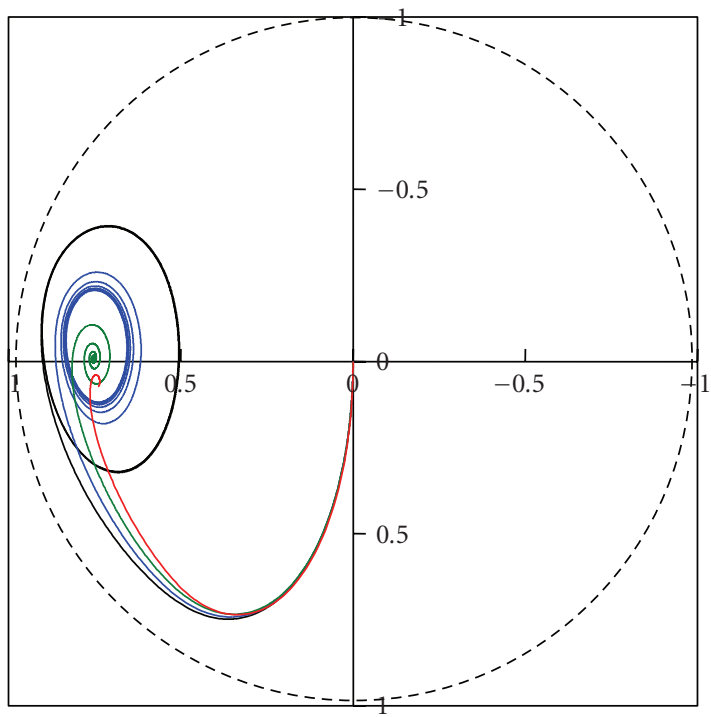

$\begin{array}{ll}-0 \mathrm{Vamp} & -40 \mathrm{~V} \mathrm{amp} \\ -30 \mathrm{Vamp} & -50 \mathrm{Vamp}\end{array}$

(c)

FIGURE 6: Quasistatic results for three-lobe bearing with squeeze-film effect, $\omega=1500 \mathrm{~Hz}, 56.65 \mathrm{~g}$ load: (a) $\Omega=320 \mathrm{rpm}$, (b) $\Omega=630 \mathrm{rpm}$, (c) $\Omega=1020 \mathrm{rpm}$. Trajectories path of rotor centre are expressed as a ration of rotor centre position over bearing clearance.

effect improves the threshold of instability by increasing the vertical eccentricity of the rotor, $\varepsilon_{Y}$.

Figures $7(a)-7(c)$ show the experimental results of the rotors' orbits obtained for rotational frequencies of $320 \mathrm{rpm}$, $630 \mathrm{rpm}$, and $1020 \mathrm{rpm}$. Their corresponding FFT responses are shown in Figures $8(a)-8(c)$. The periodic circular orbits with FFT responses showing the vibration amplitude at the same frequency as the rotational frequency are a direct result of rotor imbalance from machining tolerances. Instabilities are apparent when the rotor executes a chaotic orbit; this is shown on the FFT responses as peaks below the rotational frequency. These peaks are at frequencies about half the rotational frequency; hence instabilities of such nature are often referred as "half-frequency whirl". The results show that the size of the orbit increases as rotational frequency increases; this is again the effect of rotors imbalance. However, at rotational speed of $1020 \mathrm{rpm}$, the elliptical orbits of the rotor with larger radiuses than the bearing radial clearance suggested the rotor to bearing wall contacts in a conical whirling mode. It was found that in the attempt to 

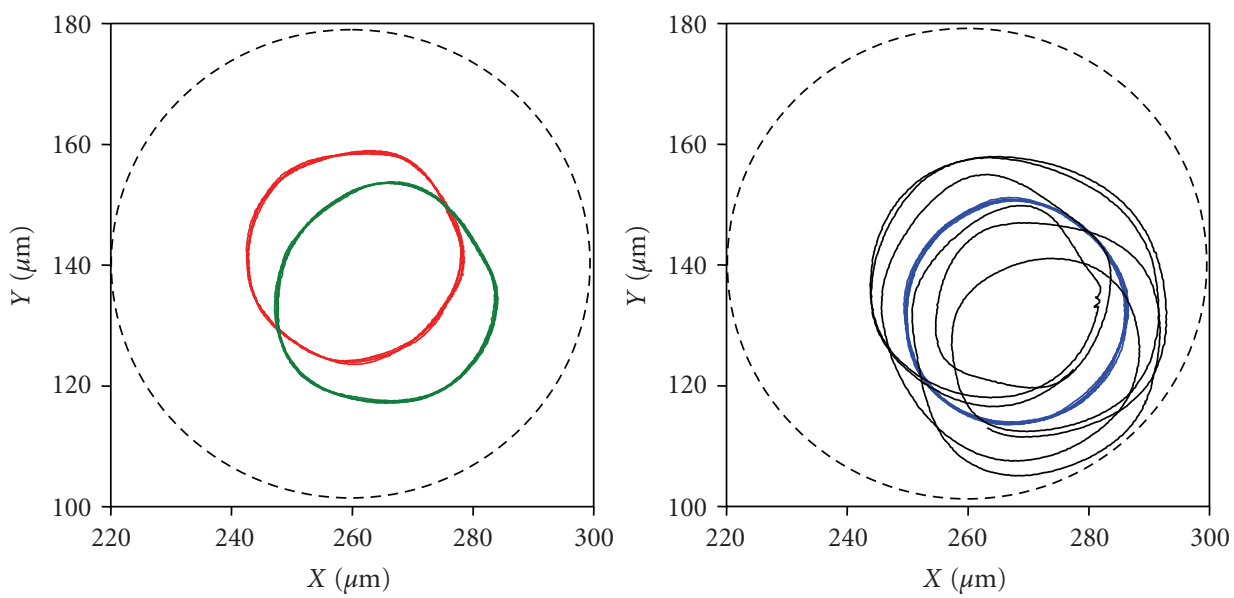

(a)
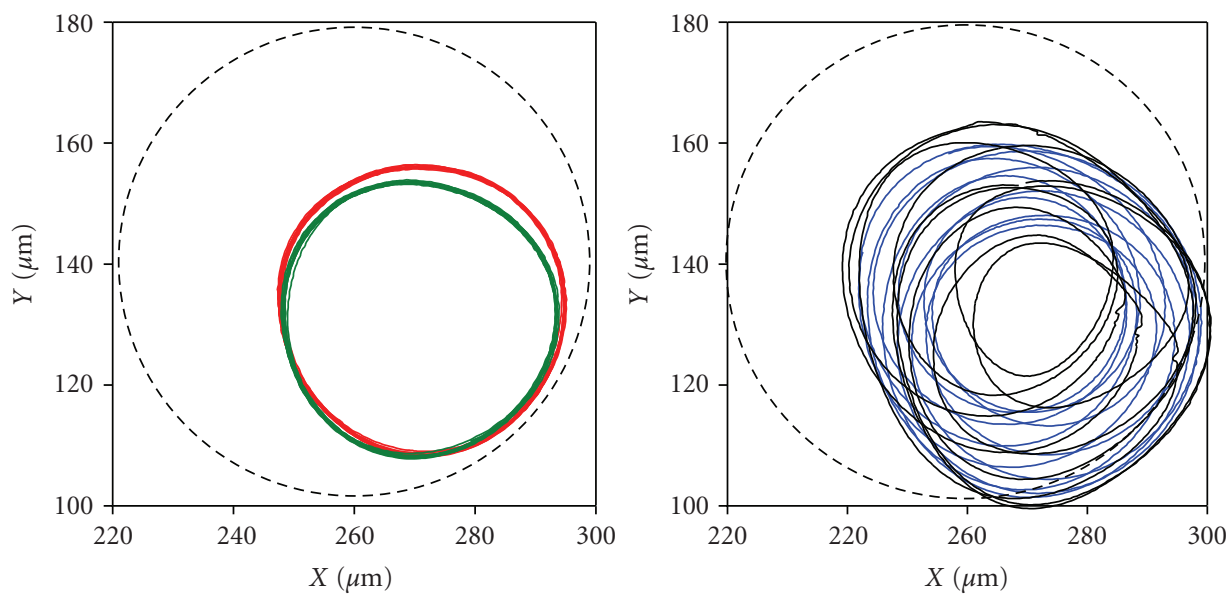

(b)
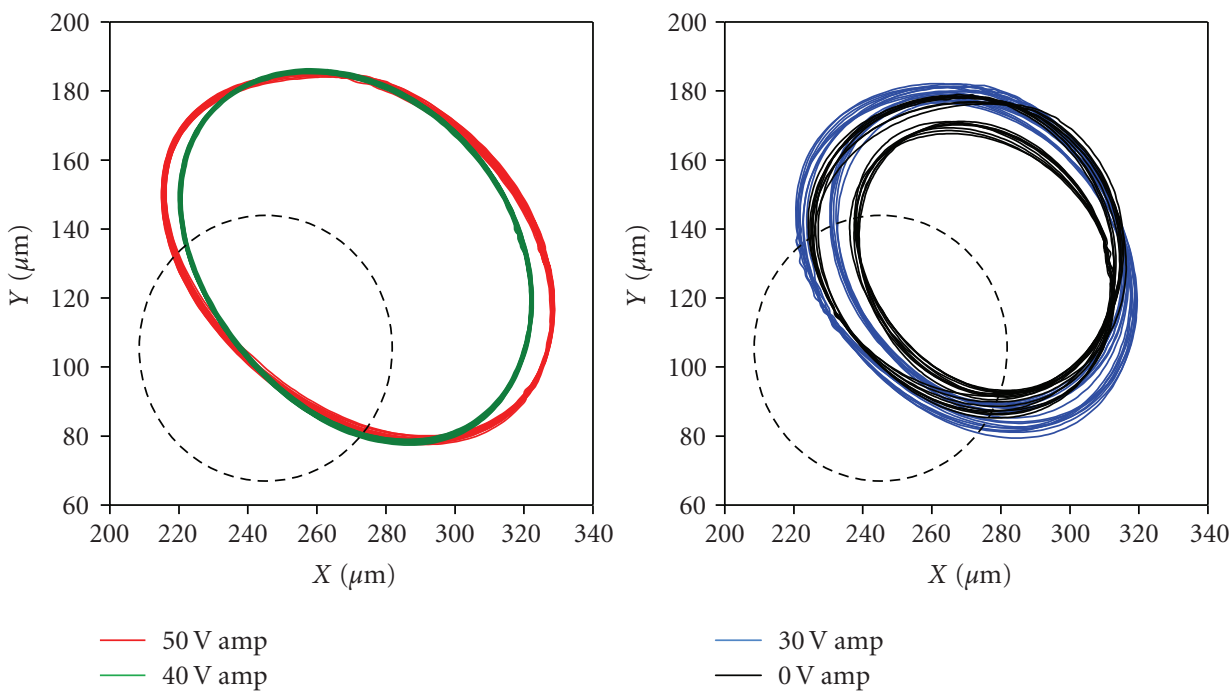

(c)

Figure 7: Rotor orbits with squeeze-film effect, $\omega=1500 \mathrm{~Hz}$, zero load: (a) $\Omega=320 \mathrm{rpm}$, (b) $\Omega=630 \mathrm{rpm}$, and (c) $\Omega=1020 \mathrm{rpm}$. 

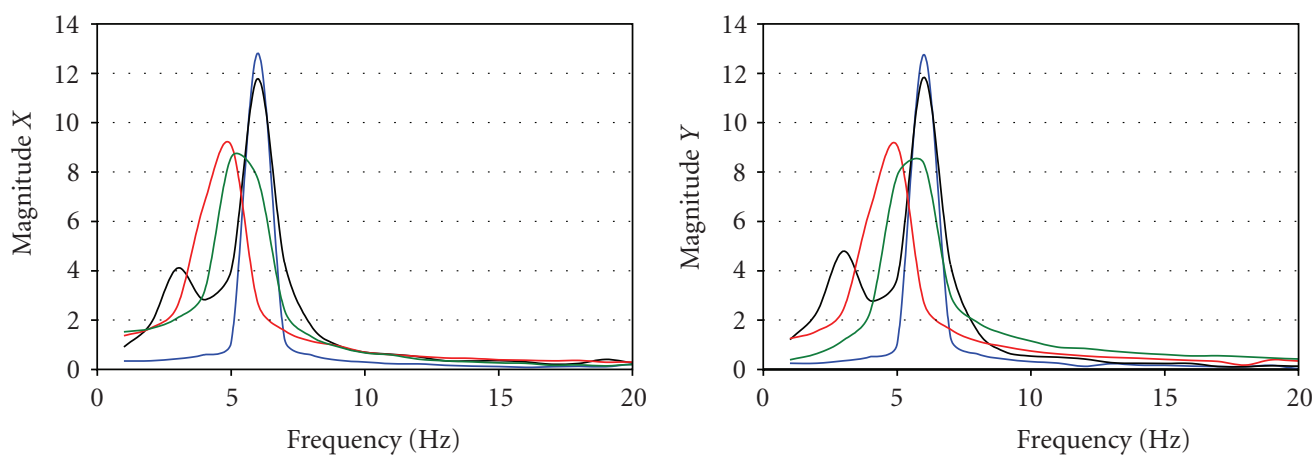

(a)
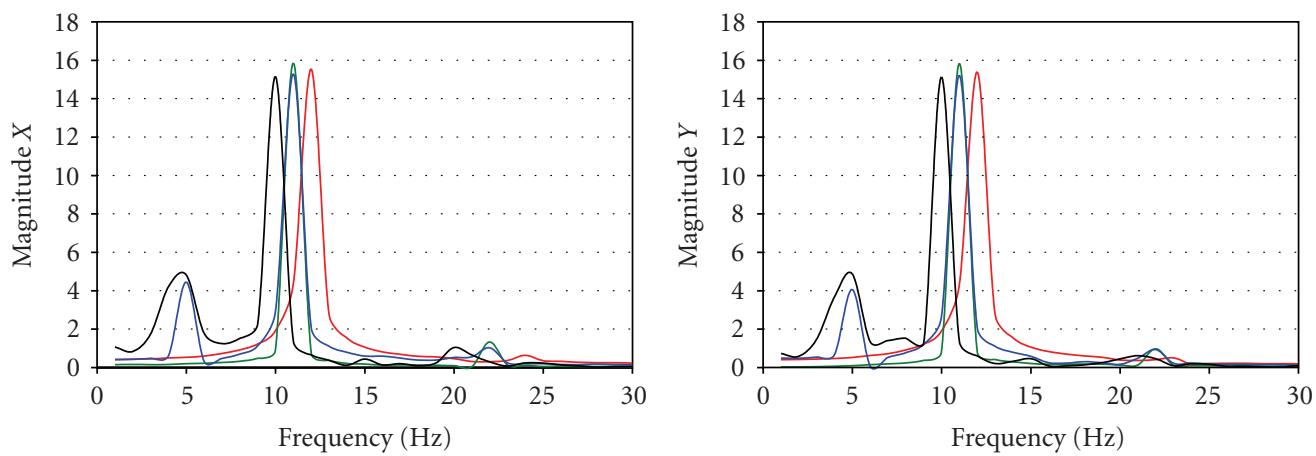

(b)
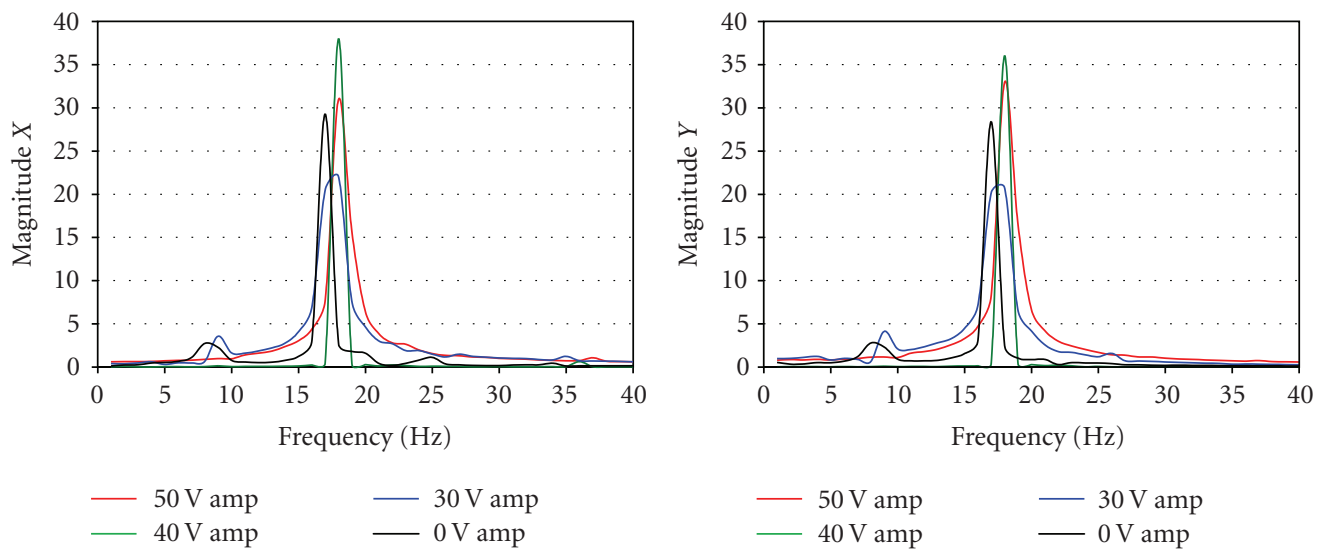

(c)

FiguRE 8: FFT responses $[\mu \mathrm{m}]$ of rotor orbits $\omega=1500 \mathrm{~Hz}$, zero load: (a) $\Omega=320 \mathrm{rpm}$, (b) $\Omega=630 \mathrm{rpm}$, and (c) $\Omega=1020 \mathrm{rpm}$.

increase the rotational speed pass this threshold, the bearing failed and the rotor was ground to a halt.

When the squeezing frequency excites a resonant frequency of the bearing, a substantial gain in the vibration response was observed. This gain in amplitudes results in increased squeeze-film effect. However, the periodic but chaotic motion generates an unsymmetrical pressure distribution, and the self-rotating effect of the rotor was observed.

\section{Concluding Remarks}

The study presented in the paper demonstrated the feasibility of a squeeze-film mechanism and the self-lifting effect by squeeze-film generated pressure. This study may be extended to improve this self-generated squeeze-film capacity of the bearing and, in turn, the threshold speed of instability will be increased. The use of ultrasonic piezoelectric elements may also be considered as this offers noise-free operation. The bearings may also be developed to create travelling waves, and the possibility of a noncontacting ultrasonic motor would also be an interesting investigation. The theoretical analysis may be extended to a full transient analysis of the squeeze-film bearing. The computational demand for this analysis is best met with the use of parallel computing. However, efforts should also be considered for improving the computational efficiency of the numerical scheme. 
The trajectory paths based on the numerical results generated by the time-marching static analysis were considered to be appropriate in assessing the dynamic stability of the bearing. Numerical results showed that the stability of the bearing could be improved by increasing the static lobe eccentricity, increasing the external load, or increasing the self-generating squeeze-film pressure. Experimental results showed that the bearing's self-generated squeeze-film pressure supporting the load on the bearing can be used during the startup phase of gas bearing operation. However, it could raise the instability threshold of the rotor up to the speed at which aerodynamic mechanism of pressure generation for a given bearing becomes active. In the case of the gas bearing, test results presented that speed was found to be $1020 \mathrm{rpm}$.

\section{Nomenclature}

c: Undeformed radial bearing clearance

$e_{X}$ : Horizontal eccentricity of rotor

$e_{Y}$ : Vertical eccentricity of rotor

$e_{W}$ : Dynamic amplitude of three-lobe motion

$e_{W 0}$ : Static amplitude of three-lobe bearing clearance

$F_{P X}$ : Dimensionless force due to pressure in the $x$ direction

$F_{P Y}$ : Dimensionless force due to pressure in the $y$ direction

$\bar{F}_{P X}$ : Time averaged dimensionless force due to pressure in the $x$ direction

$\bar{F}_{P Y}$ : Time averaged dimensionless force due to pressure in the $y$ direction

g: Acceleration due to gravity

$h: \quad$ Film thickness

$l_{0}$ : Bearing axial length

$m_{\alpha}$ : Mass of load

$m_{0}:$ Mass of shaft

$M_{0}$ : Dimensionless mass (see (13a))

$p_{0}$ : Ambient pressure

$p: \quad$ Film pressure

$P: \quad$ Film dimensionless pressure, $P=p / p_{a}$

$r_{0}:$ Radius of rotor

$t$ : Time

T: $\quad$ Dimensionless time, $T=\Omega t$

$z: \quad$ Length displacement

$Z$ : Dimensionless bearing length displacement, $Z=z / l_{0}$

$\alpha$ : Tilting angle

$\varepsilon_{\mathrm{WA}}$ : Dimensionless dynamic amplitude of three-lobe motion, $\varepsilon_{\mathrm{WA}}=e_{\mathrm{WA}} / c$

$\varepsilon_{\mathrm{WO}}$ : Dimensionless static amplitude, $\varepsilon_{\mathrm{WO}}=e_{\mathrm{WO}} / c$

$\varepsilon_{X}$ : Horizontal eccentricity ratio of rotor, $\varepsilon_{X}=e_{X} / c$

$\varepsilon_{Y}$ : Vertical eccentricity ratio of rotor, $\varepsilon_{Y}=e_{Y} / c$

$\varepsilon_{X 0}$ : Horizontal eccentricity ratio of rotor at previous time step

$\varepsilon_{Y 0}$ : Vertical eccentricity ratio of rotor at previous time step

$\theta: \quad$ Angular length of film

$\mu$ : $\quad$ Viscosity of fluid film

$\tau: \quad$ Dimensionless time, $\tau=\omega t$

$\omega: \quad$ Frequency of the three-lobe motion

$\Omega$ : Rotational frequency of rotor.

\section{References}

[1] D. B. Reynolds and W. A. Gross, "Experimental investigation of whirl in self-acting air-lubricated journal bearings," ASLE Transactions, vol. 5, pp. 392-403, 1962.

[2] J. K. Park and K. W. Kim, "Stability analyses and experiments of spindle system using new type of slot-restricted gas journal bearings," Tribology International, vol. 37, no. 6, pp. 451-462, 2004.

[3] F. K. Choy, M. J. Braun, and Y. Hu, "Nonlinear effects in a plain journal bearing. Part 1. Analytical study," Journal of Tribology, vol. 113, no. 3, pp. 555-562, 1991.

[4] J. W. Lund, "Review of the concept of dynamic coefficients for fluid film journal bearings," Journal of Tribology, vol. 109, no. 1, pp. 37-41, 1987.

[5] V. Castelli and H. G. Elrod, "Solution of the stability problem for 360 deg self-acting, gas-lubricated bearings," ASME Journal of Basic Engineering, vol. 87, pp. 199-212, 1965.

[6] L. X. Liu and Z. S. Spakovszky, "Effects of bearing stiffness anisotropy on hydrostatic micro gas journal bearing dynamic behavior," Journal of Engineering for Gas Turbines and Power, vol. 129, no. 1, pp. 177-184, 2007.

[7] C. J. Teo, Z. S. Spakovszky, and S. A. Jacobson, "Unsteady flow and dynamic behavior of ultrashort Lomakin gas bearings," Journal of Tribology, vol. 130, no. 1, Article ID 011001, 2008.

[8] D. N. Ha, T. A. Stolarski, and S. Yoshimoto, "An aerodynamic bearing with adjustable geometry and self-lifting capacitypart 1: self-lift capacity by squeeze film," Proceedings of the Institution of Mechanical Engineers J, vol. 219, no. 1, pp. 33-39, 2005. 

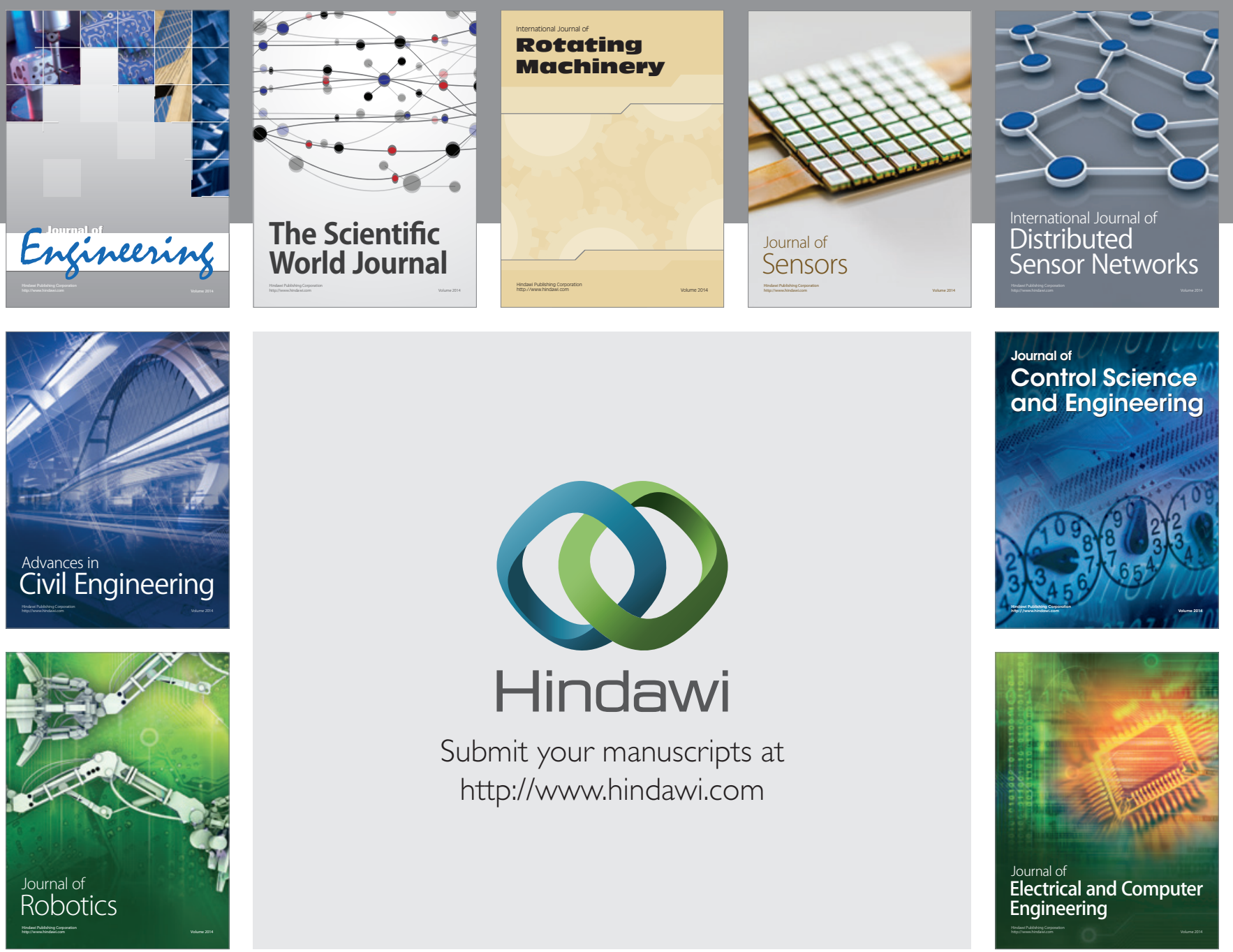

Submit your manuscripts at

http://www.hindawi.com
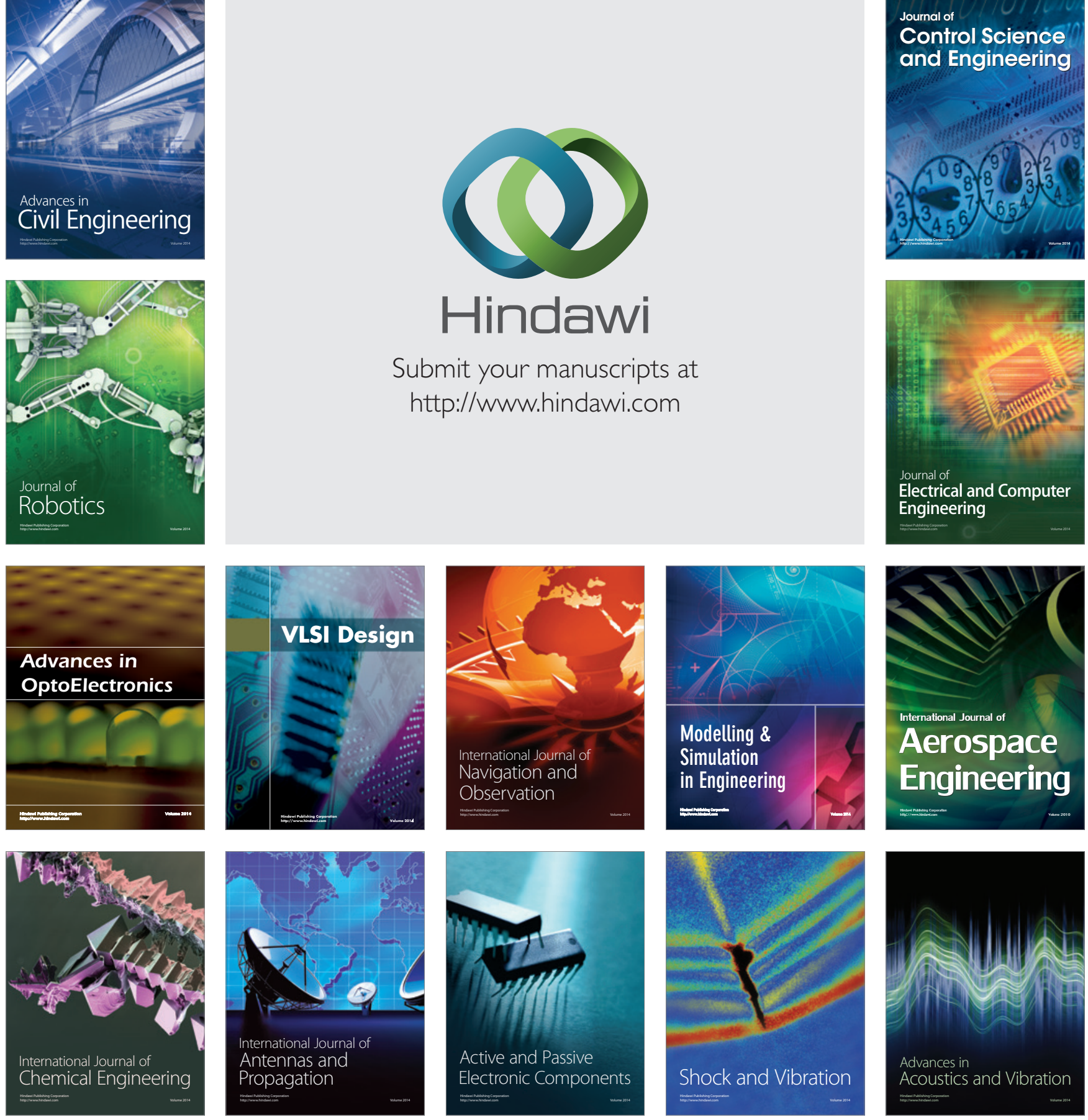